\title{
Universality of Oscillation Theory Laws. Types and Role of Mathematical Models
}

\author{
POLINA S. LANDA \\ Department of Physics, Moscow State University, Moscow 119899, Russia
}

(Received 6 December 1996)

\begin{abstract}
The universality of oscillation theory laws is discussed. It is suggested that all models of concrete systems be separated into four categories: models - "portraits" of investigated systems, models of the type of "black box", aggregating models, and models of certain phenomena which can occur in real systems. As an example of the model of the fourth type, the equation of oscillations of a pendulum with a randomly vibrating suspension axis is considered for the purpose of clarifying the mechanism of the transition to turbulence.
\end{abstract}

Keywords: Oscillations, Turbulence, Noise-induced phase transitions

\section{INTRODUCTION}

The theory of oscillations is the science that studies oscillatory motions irrespective of their physical nature. By oscillatory motions are meant any limited changes of body state taking place in a long time interval. Then, because these changes are limited, they must necessarily be "hither and thither" [1]. Such a definition of the theory of oscillations is very common. We know that other sciences study changes of state of bodies too. How does the theory of oscillations differ from them? An answer to this question has been given by Mandelshtam [1]. Contrary to other sciences for which the prime interest is in what happens to a body at a given instant, the theory of oscilla- tions concerns "the general character of a process taken as a whole over a long interval of time".

\section{THE UNIVERSALITY OF OSCILLATION THEORY LAWS}

In common with any other science the theory of oscillations has laws of its own. It is essentially that these laws are general and not depending on the concrete type of the system studied. Unfortunately, these general laws up till now are not well-formulated. However, many of the specialists in the oscillation theory use, sometimes intuitively, these laws in their studies. By using the knowledge of the general laws one can profitably 
predict different phenomena from diversified areas of science. The discovery of light combination scattering effect ${ }^{1}$ by Mandelshtam [2] is a typical example of such a prediction. As for the analogy between light combination scattering and the usual objects of oscillation theory, Mandelshtam wrote [3], "From the point of view of the theory of oscillations, wireless telephony and light combination scattering are the same. It is modulation. Sound - in radio, and atomic oscillations-in combination scattering". Thus, one of the general laws of the oscillation theory, which implies that in any nonlinear system modulation is possible, was the basis for Mandelshtam's prediction. Another example of the general laws of the oscillation theory is the possibility of synchronization of any self-oscillatory system by means of the corresponding external action with different mechanisms of the synchronization in the cases of slight and strong actions [4-6]. As a further example we can point to the possible routes for the appearance of chaos and stochasticity in any selfoscillatory systems [7].

The knowledge of the general laws of the oscillation theory is also important in that for study of any oscillatory phenomena one can use simple models and be sure that in concrete more complicated systems these phenomena will be also of the same character. This fact is used tacitly by all researchers.

It is evident that the general laws of the oscillation theory must manifest themselves identically in systems of diversified physical nature. Regarding this, Mandelshtam told students in one of his lectures on the theory of oscillations, "All of you know such systems as a pendulum and an oscillatory circuit, and also know that from the oscillatory point of view they are similar. Now all this is trivial, but it is wonderful that this is trivial." These ideas have not yet become fashionable. In the paper "L.I. Mandelshtam and the theory of non-linear oscillations" Andronov [8] wrote of the lectures and seminars of Mandelshtam,
"Lectures and seminars of Mandelshtam have sometimes contained new scientific results which were not published. But, perhaps the greatest significance of these lectures lies in the methodical inculcating of habits of oscillatory thinking, in the general rise of the oscillatory culture". Unfortunately, many even prominent scientists, studying concrete problems, are still lacking in "oscillatory culture". This is the main cause of the fact that up to the present the scientific works, which are absolutely erroneous from the point of view of the general laws of the oscillation theory, occur from time to time.

There is much speculation that the generality of the laws of the oscillation theory is based on the similarity of the equations describing oscillatory processes in different systems. Every so often this is so indeed. However, the laws mentioned above (and many other ones) are independent of the concrete form of the equations. It is only sufficient that the equations belong to a certain class, which in common is moderately broad.

\section{TYPES OF MATHEMATICAL MODELS AND THEIR ROLE}

The availability of analogies between oscillatory processes in systems of diversified physical nature is why the theory of oscillations got its subject of investigation, and thereby took the status of an original science. A dynamical system is such a subject $[9,10]$. A dynamical system is a system whose behavior is predetermined by a set of rules (algorithm). In particular, and most frequently, the behavior of a dynamical system is described by differential, integral or finite-difference equations. Obviously, a dynamical system is a model of a real system. So we can say that the theory of oscillations studies abstract models, but not concrete systems. These models can be conventionally separated into four categories: models "portraits" of investigated systems, models of the

\footnotetext{
${ }^{1}$ In western literature this effect is usually called the Raman effect.
} 
type of "black box", aggregating models, and models of certain phenomena which can occur in real systems.

The models of the first type are constructed on the basis of the description, as more detailed as possible, of all elements of the system studied. Such models, e.g., are studied in physics. An example is the Navier-Stokes equations and their finite-difference approximations describing fluid motion with a high degree of accuracy. As another example we can point to certain models of heart activity or breathing [11]. These models, as a rule, are very complicated and cumbersome for detailed analysis and revealing principal features of system's behavior.

When the models of the second type are constructed, the system studied is considered as a certain "black box" with given inputs and the outputs which are able to be measured. Further, a model, as simpler as possible, is chosen. The free parameters of this model are determined from the conditions of the minimum, according to a given criterion, of the difference between the model and the system outputs for identical inputs. A number of examples of such models for chemical reactions is given in [12]. The same approach is conceptually used for reconstruction a model from experimental data (see, for example, [13]).

The models of the third type are constructed on the basis of the analysis of aggregate behavior of individual elements of the system studied. A classical example of the models of this type is the model "prey-predator" by Lotka-Volterra $[14,15]$. The other interesting examples are the model of an immune reaction illustrating an oscillatory course of some chronic diseases $[16,10]$ and the model of the economic progress of human society $[17,10]$.

Finally, the models of the fourth type are constructed for analysis of a certain phenomenon no matter in what system it occurs. For example, the phenomenon of self-excitation of selfoscillations can be modeled by the van der Pol equation; the phenomenon of synchronization of periodic self-oscillations can be also modeled by the van der Pol equation but with an external force; oscillatory character certain chemical reactions is demonstrated by the models "brusselator" and "oregonator"; the transition to chaos via an infinite period-doubling bifurcation sequence can be modeled by a logistic map [18]; the transition to chaos via intermittency can be modeled by a map of the form [7]

$$
x_{n+1}=x_{n}+a x_{n}^{z},
$$

where $z$ is an even number, $a$ is the parameter; some processes in continuous media can be modeled by coupled map lattices [19]. Similar examples can be given in abundance. It should be particularly emphasized that the mere possibility and utility of such models are based on the universality of the oscillation theory laws.

\section{THE TRANSITION TO TURBULENCE AND OSCILLATIONS OF A PENDULUM CAUSED BY RANDOM VIBRATION OF ITS SUSPENSION AXIS}

In this section we dwell more closely on a model of the transition to turbulence in the form of the equation of oscillations of a pendulum with a randomly vibrating suspension axis [20]. In [21, $22,10]$ we hypothesized that turbulent processes in nonclosed fluid flows are not self-oscillations but are caused by fluctuations both internal (natural) and external (technical). This hypothesis is based on the fact that instability of nonclosed laminar flows is convective. By this is meant that a small disturbance being given at some point of the flow will not indefinitely increase with time but will drift downstream. It follows from this property of convectively unstable systems that they are not self-oscillatory systems, but only amplifiers of disturbances. To make these systems self-oscillatory it is necessary to form feedback. However, in nonclosed flows such feedback is absent, i.e. for sufficiently large Reynolds' numbers such flows are amplifiers of fluctuations. 
Because the turbulent processes closely resemble self-oscillations in appearance, we further suggested that owing to the amplified fluctuations a certain phase transition can occur in the fluid flow and, as a result, the flow transforms into a qualitatively new state associated with the excitation of noise-induced self-oscillations. It can be believed that the onset of turbulence, which is characterized by presence of large-scale, comparatively regular, structures against a background of fine-scale random motions, is just such a transition. To make sure that such a transition is possible in principle, we studied, both analytically and numerically, a simple model in the form of the equation for oscillations of a pendulum with a randomly vibrating suspension axis $[23,24]$.

\subsection{Noise-Induced Oscillations of a Pendulum with a Vibrating Suspension Axis}

The equation studied is

$$
\ddot{\varphi}+2 \beta\left(1+\alpha \dot{\varphi}^{2}\right) \dot{\varphi}+\omega_{0}^{2}(1+\xi(t)) \sin \varphi=0,
$$

where $\varphi$ is the pendulum's angular deviation from the equilibrium position, $2 \beta\left(1+\alpha \dot{\varphi}^{2}\right) \dot{\varphi}$ is the value proportional to the moment of the friction force which is assumed to be nonlinear, $\omega_{0}$ is the natural frequency of small pendulum's oscillations, and $\xi(t)$ is the acceleration of the suspension axis that is a comparatively wideband random process with nonzero power spectrum density $\kappa(\omega)$ at the frequency $\omega=2 \omega_{0}$.

Setting $\varphi \approx A \cos \left(\omega_{0} t+\phi\right)$ and solving Eq. (1) approximately by the Krylov-Bogolyubov method we obtain the following truncated equations for the amplitude $A$ and phase $\phi$ of the oscillations $[23,24]$ :

$$
\begin{aligned}
& \dot{A}=\frac{\omega_{0}^{2} K_{1}}{4}\left(\eta-\frac{3 \beta \tilde{\gamma}}{\omega_{0}^{2} K_{1}} A^{2}\right) A+\frac{\omega_{0}}{2} A \zeta_{1}(t), \\
& \dot{\phi}=\omega_{0} M-\frac{3}{8} \omega_{0} \gamma A^{2}+\omega_{0} \zeta_{2}(t),
\end{aligned}
$$

where

$$
\eta=1-\frac{4 \beta}{\omega_{0}^{2} K_{1}}
$$

is the parameter characterizing the extent to which the noise intensity is in excess of its critical value for which the phase transition arises, $\tilde{\gamma}=\gamma+\alpha \omega_{0}^{2}, \gamma$ is the coefficient of the first nonlinear term in the expansion of $\sin \varphi, K_{1}=$ $\kappa\left(2 \omega_{0}\right) / 2$ is the parameter characterizing the intensity of suspension axis' vibration, $\zeta_{1}(t)$ is a random process with zero mean value and the intensity $K_{1}, M=\left\langle\overline{\xi \cos ^{2} \psi}\right\rangle$, and $\zeta_{2}(t)$, much like to $\zeta_{1}(t)$, is a random process with zero mean value and the intensity $K_{2}=\left(\kappa(0)+K_{1}\right) / 4$.

The Fokker-Planck equation associated with Eqs. (2) is

$$
\begin{aligned}
& \frac{\partial w(A, \phi)}{\partial t} \\
& =-\frac{\partial}{\partial A}\left(\left(\frac{\omega_{0}^{2} K_{1}}{4} \eta-\frac{3}{4} \beta \tilde{\gamma} A^{2}\right) A w(A, \phi)\right) \\
& \quad-\omega_{0}\left(\frac{3}{8} \gamma A^{2}-M\right) \frac{\partial w(A, \phi)}{\partial \phi} \\
& +\frac{K_{1} \omega_{0}^{2}}{8} \frac{\partial^{2}}{\partial A^{2}}\left(A^{2} w(A, \phi)\right)+\frac{K_{2} \omega_{0}^{2}}{2} \frac{\partial^{2} w(A, \phi)}{\partial \phi^{2}} .
\end{aligned}
$$

Finding from this equation the steady-state probability density for the amplitude $A$, we can calculate the mean value of the steady-state amplitude. It is

$$
\langle A\rangle= \begin{cases}\sqrt{\frac{\omega_{0}^{2} K_{1}}{3 \beta \tilde{\gamma}}} \frac{\Gamma(\eta+1 / 2)}{\Gamma(\eta+1)} \eta & \text { for } \eta \geq 0, \\ 0 & \text { for } \eta \leq 0 .\end{cases}
$$

It follows from this that for $\eta>0$ the parametric excitation of pendulum's oscillations occurs under the effect of noise. This manifests itself in the fact that the mean value of the amplitude becomes different from zero.

Numerical simulation of Eq. (1) showed that, as one would expect from the theoretical results, 
when the intensity of the suspension axis vibration, characterized by the value of $\kappa\left(2 \omega_{0}\right)$, is in excess of a certain critical value $\kappa_{\mathrm{cr}}$ proportional to the friction factor $\beta$, the excitation of pendulum's oscillations occurs that makes itself evident in the fact that the variance of the pendulum's angular deviation becomes nonzero. This phenomenon can be considered as a noise-induced phase transition and the birth of an induced attractor. The latter follows from the fact that the correlation dimension of the attractor constructed from the data obtained by the numerical simulation with using Takens' technique turns out to be finite $[23,24]$. However this attractor is very noisy. This is evidenced by the fact that the embedding dimension calculated by both well-adapted basis technique [25] and the method of BroomheadKing [26] is very large.

Examples of the noise-induced oscillations and the dependence of the variance of the pendulum's angular deviation on the relative power spectrum density $\kappa\left(2 \omega_{0}\right) / \kappa_{\mathrm{cr}}$ found by numerical simulation of Eq. (1) are depicted in Fig. 1. It is seen from this figure that close to the excitation threshold the pendulum's oscillations possess the property of a peculiar kind of intermittency, ${ }^{2}$ i.e. over prolonged periods the pendulum oscillates in the immediate vicinity of its equilibrium position (socalled "laminar" phases); these slight oscillations alternate with short strong bursts ("turbulent" phases). Away from the threshold the duration of laminar phases decreases and of turbulent increases, and laminar phases ultimately disappear. The variance of the pendulum's angular deviation increases in the process. We emphasize that turbulence for transient Reynolds numbers exhibit also this property [29-31]. It is no chance that the first theoretical works concerning the intermittency phenomenon were made by the specialists in the field of turbulence [32].

Interestingly enough that in the case of the pendulum the intermittency observed is different
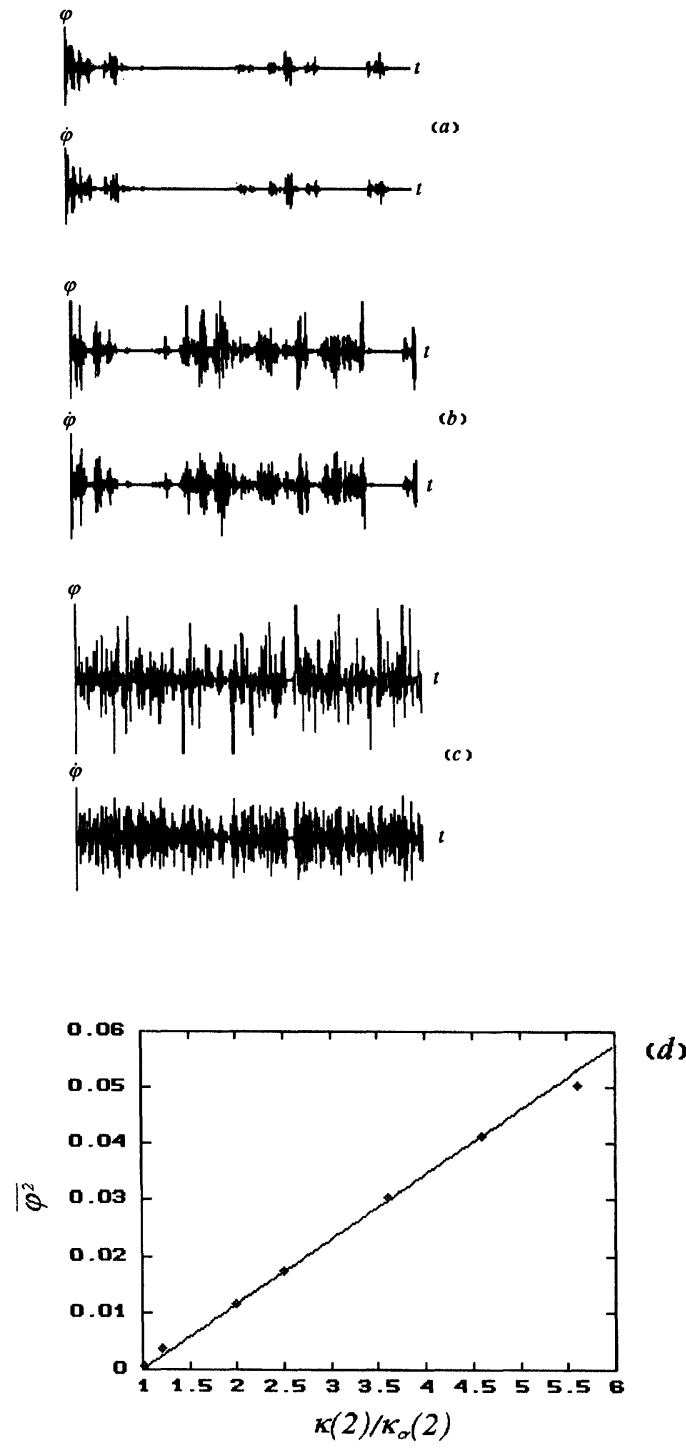

FIGURE 1 The dependencies $\varphi(t)$ and $\dot{\varphi}(t)$ for $\omega_{0}=1$, $\beta=0.1, \alpha=100$ (a) $\kappa(2) / \kappa_{\mathrm{cr}}=1.01$, (b) $\kappa(2) / \kappa_{\mathrm{cr}}=1.2$, and (c) $\kappa(2) / \kappa_{\mathrm{cr}}=5.6$; (d) the dependence of $\overline{\varphi^{2}}$ on $\kappa(2) / \kappa_{\mathrm{cr}}$ (the straight line $\overline{\varphi^{2}}=0.01151\left(\kappa(2) / \kappa_{\mathrm{cr}}-1\right)$ is shown as a solid line).

from all types of the intermittency described in $[27,7,28]$, although they are similar in their external manifestations. It is so called "on-off-intermittency". ${ }^{3}$ The term "on-off-intermittency" was

\footnotetext{
${ }^{2}$ The intermittency is described, for example, in [27,7,28].

${ }^{3}$ Author is indebted to S.P. Kuznetsov and A. Cenys for calling his attention to this fact.
} 
recently introduced by Platt et al. [33], though a map associated with the similar type of intermittent behavior was first considered by Pikovsky [34]. It is essential that this type of intermittency can occur not only in dynamical systems but in stochastic systems as well [35]. In [35] the statistical properties of on-off intermittency were found from the analysis of the map

$$
x_{n+1}=a\left(1+z_{n}\right) x_{n}+f\left(x_{n}\right),
$$

where $z_{n}$ is either a certain deterministic chaotic process or a random process, $a$ is the bifurcation parameter, $f\left(x_{n}\right)$ is a nonlinear function. It was obtained that the mean duration of laminar phase has to be proportional to $a^{-1}$.

Let us calculate the mean duration of laminar phase using Eqs. (2) and the Fokker-Planck equation (3) associated with them. We shall assume that the pendulum oscillates in laminar phase if the oscillation amplitude $A$ is no more than a certain value $\epsilon$. Then the mean duration of the laminar phase $\tau_{\epsilon}$ will be determined by the mean duration of random walk of the representative point inside the circle of radius $\epsilon$ on the plane $\varphi, \dot{\varphi}$. This duration can be calculated (see [7]) using the steady-state solution of Eq. (3) with the boundary condition

$$
\left.w(A, \phi)\right|_{A=\epsilon}=0 .
$$

Because the value of $\epsilon$ is assumed to be small, we can neglect the term (3/4) $\beta \tilde{\gamma} A^{2}$ in Eq. (3). In so doing the solution of Eq. (3) with the boundary condition (4) is

$$
w(A, \phi)=\frac{8 G_{0}}{\omega_{0}^{2} K_{1}(1-2 \eta) A}\left(\epsilon^{1-2 \eta} A^{2 \eta-1}-1\right),
$$

where $G_{0}$ is the value of the probability flow

$$
G=\frac{\omega_{0}^{2} K_{1}}{4}\left(\eta A w-\frac{1}{2} \frac{\mathrm{d}}{\mathrm{d} A}\left(A^{2} w\right)\right)
$$

across any circumference inside the circle of radius $\epsilon$. The value $G_{0}$ is determined from the normal- ization condition by integrating the expression (5) over the circle of radius $\epsilon$. Using this condition and taking into account that $\tau_{\epsilon}=2 G_{0}^{-1}$ [36] we obtain

$$
\tau_{\epsilon}=\frac{16 \pi \epsilon}{\omega_{0}^{2} K_{1} \eta}
$$

It follows from this that for small $\eta$ the mean duration of the laminar phase is inversely proportional to $\eta$. This result agrees with [35].

If the intensity of the suspension axis random vibration is under its threshold value then the excitation of pendulum's oscillations can be initiated by small additional low-frequency vibration of the suspension axis. The inclusion of this vibration can be carried out by substitution into Eq. (1) of $\xi+a \cos \omega_{a} t$ in place of $\xi$, where $a$ and $\omega_{a}$ are, respectively, the amplitude and frequency of the additional vibration of the suspension axis. The results of numerical simulation of Eq. (1) for different values of $a$ are represented in Fig. 2. We see that the excitation of oscillations, as the amplitude $a$ increases, is of a threshold character. For $\omega_{a}=0.318, \kappa\left(2 \omega_{0}\right) / \kappa_{\mathrm{cr}}=0.51$ the threshold value of $a$ is equal to 1.1. The dependence of the pendulum's angular deviation mean square on the difference between the amplitude $a$ and its threshold value is found to be close to linear (see Fig. 3(a)). For $a>a_{\mathrm{cr}}$ the oscillations excited are not distinguished from those excited due to random vibration only. The oscillation intensity is greater, the larger the $a$. By this is meant that the low-frequency vibration initiates the noise-induced phase transition and the birth of the induced attractor. In the case when the intensity of the suspension axis random vibration is in excess of its threshold value additional low-frequency vibration contributes significantly to the intensification of noise-induced oscillations. The dependence of $\bar{\varphi}^{1 / 2}$ on $a$ for $\kappa(2) / \kappa_{\mathrm{cr}}(2)=2.23, \omega_{a}=1.5$ is given in Fig. 3(b).

Let us consider now a possibility of suppressing noise-induced pendulum's oscillations by high-frequency harmonic action. Numerical simulation of Eq. (1) with $\xi+a \cos \omega_{a} t$ in place of $\xi$, 


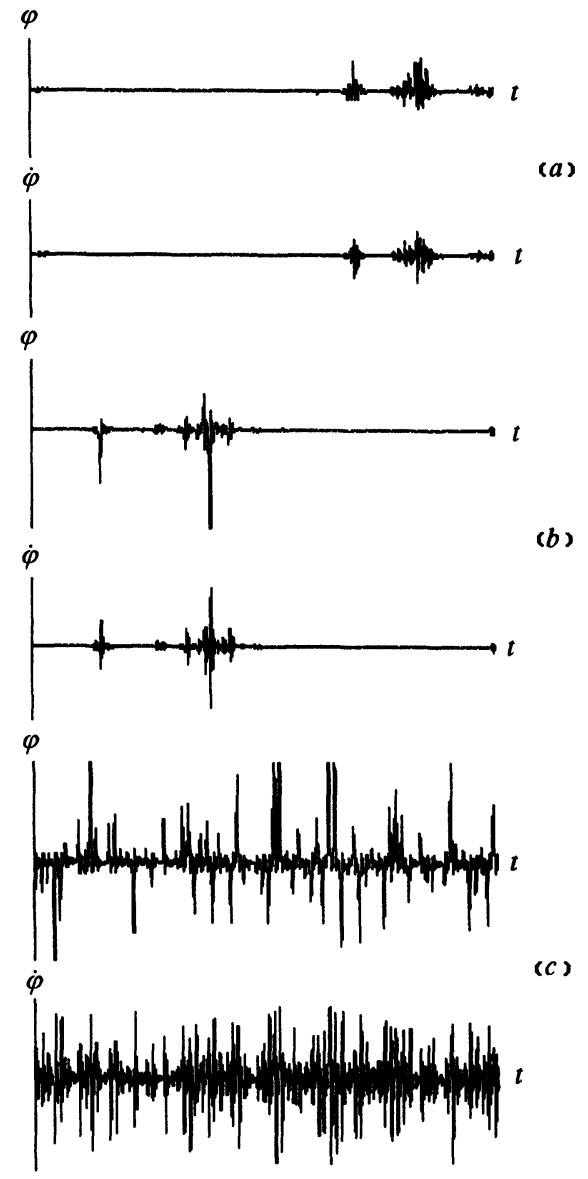

FIGURE 2 The dependencies $\varphi(t)$ and $\dot{\varphi}(t)$ for $\omega_{0}=1$, $\beta=0.1, \alpha=100, \kappa\left(2 \omega_{0}\right) / \kappa_{\mathrm{cr}}=0.51, \omega_{a}=0.318$ (a) $a=1.12$, (b) $a=1.2$, and (c) $a=1.5$. where $\omega_{a}>2 \omega_{0}$, shows that such suppression does occur. The results of the simulation for sufficiently large value of $\omega_{a}$ are represented in Fig. 4. It is seen from this figure that, for small amplitudes of the high-frequency action, the action has little or no effect on the noise-induced oscillations existing (see Fig. 4(a). As the amplitude increases the intensity of the noise-induced oscillations decreases rapidly and the duration of "laminar" phases increases in the process (see Fig. 4(b-d). When the amplitude is in excess of a certain critical value (for the case considered it is equal to 42) the oscillations are suppressed entirely. As the amplitude increases further the oscillations arise again, but because the conditions of the corresponding parametric resonance come into play. If the frequency of the additional action is not-too-high then the perfect suppression does not occur: as the action amplitude increases the intensity of the noise-induced oscillations first decreases to a certain minimal value, which is smaller the greater the action frequency, and then increases. True, this minimal value is attained for the larger amplitudes of the action, the higher its frequency. The aforesaid is illustrated in Fig. 5.

We emphasize that the initiation and suppression of noise-induced pendulum's oscillations occur by means not only of additional parametric action, but of additional additive action as well.

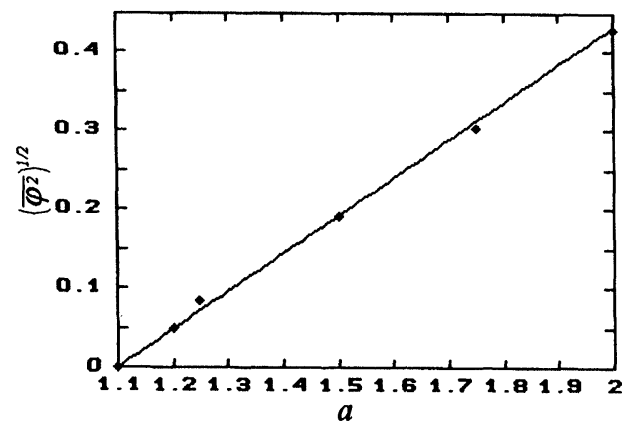

(a)

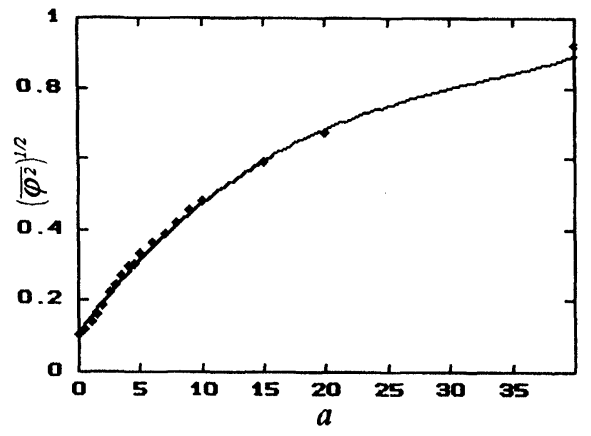

(b)

FIGURE 3 The dependencies of $\bar{\varphi}^{2} 1 / 2$ on $a$ for $\omega_{0}=1, \beta=0.1, \alpha=100$ (a) $\kappa\left(2 \omega_{0}\right) / \kappa_{\mathrm{cr}}=0.51, \omega_{a}=0.318$ (the straight line ${\overline{\varphi^{2}}}^{1 / 2}=0.48(a-1.1)$ is shown as a solid line $),(\mathrm{b}) \kappa\left(2 \omega_{0}\right) / \kappa_{\mathrm{cr}}=2.23, \omega_{a}=1.5$. 

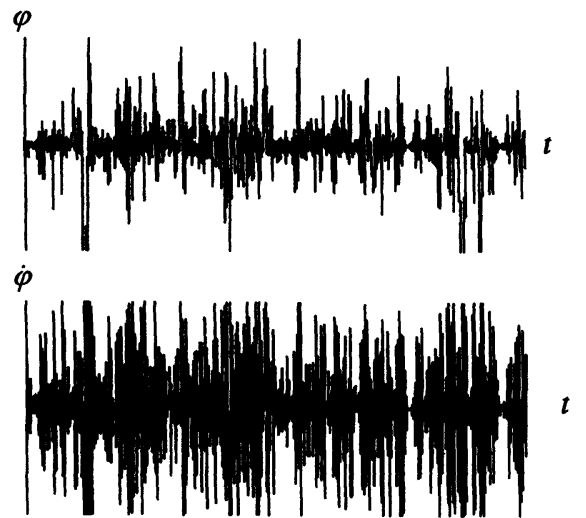

(a)
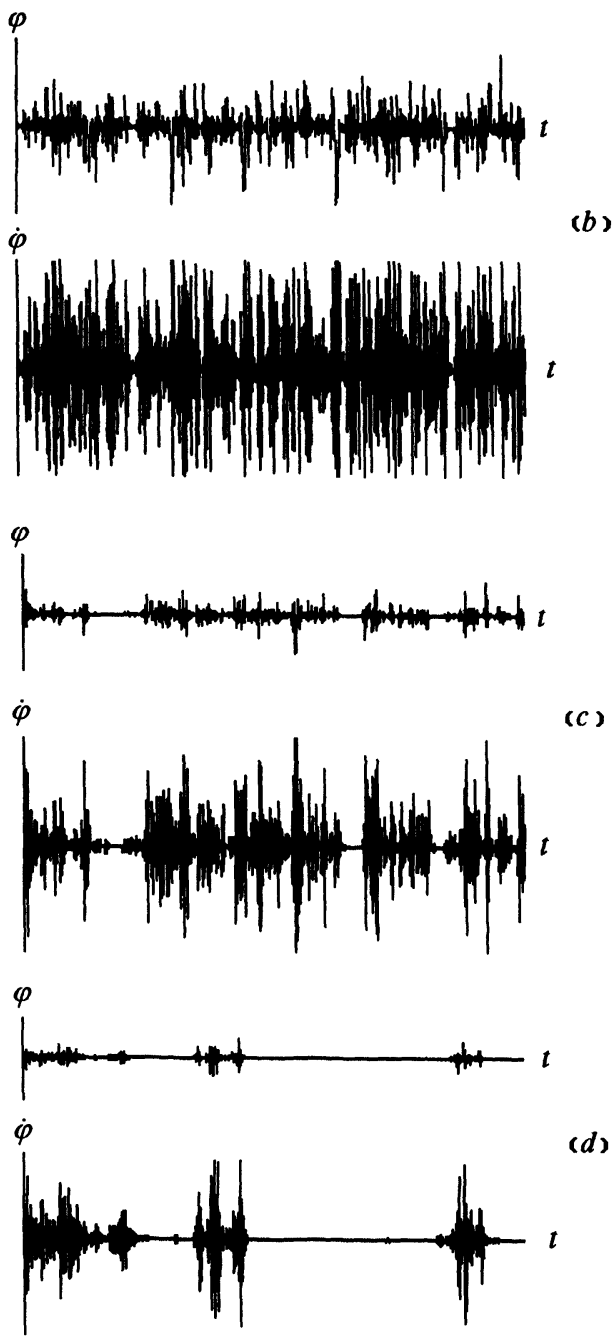

FIGURE 4 The dependencies of $\varphi(t)$ and $\dot{\varphi}(t)$ for $\omega_{0}=1$, $\beta=0.1, \alpha=100, \kappa\left(2 \omega_{0}\right) / \kappa_{\mathrm{cr}}=5.6, \omega_{a}=19.757$ (a) $a=5$, (b) $a=15$, (c) $a=30$, and (d) $a=40$.

\subsection{The Comparison of the Results of the Study of Pendulum's Oscillations with a Number of Numerical and Real Experiments Concerning Turbulence in Pipes and Free Jets}

A number of numerical and real experiments concerning turbulence shows that there exist the profound parallels between turbulent processes and noise-induced pendulum's oscillations. These parallels reinforce our hypothesis about fluctuational origin of turbulence.

One of the indications that turbulence is not a self-oscillatory process is a numerical experiment performed by Nikitin [37] which relate to the simulation of turbulent flow in pipes of a finite length. He studied fluid flow in a circular pipe of radius $R$ with a given velocity at the input crosssection and with the so-called soft boundary conditions at the output cross-section; these latter are

$$
\frac{\partial^{2} u}{\partial x^{2}}=\frac{\partial^{2} \xi}{\partial x^{2}}=\frac{\partial^{2} \eta}{\partial x^{2}}
$$

where $u$ is the longitudinal component of the flow velocity, $\xi$ and $\eta$ are, respectively, the radial and azimuth components of the vorticity $\Omega=\operatorname{rot} \mathbf{V}$, $\mathbf{V}=\{u, v, w\}$ is the vector of the flow velocity in cylindrical coordinates $x, r, \theta$. The components of the flow velocity at the pipe input cross-section were assigned in the form

$$
\begin{aligned}
u & =u_{0}\left(1-\frac{r^{2}}{R^{2}}\right)+A \operatorname{Re}\left(u^{\prime}(r) \mathrm{e}^{-\mathrm{i} \omega t}\right) \cos \theta, \\
v & =A \operatorname{Re}\left(v^{\prime}(r) \mathrm{e}^{-\mathrm{i} \omega t}\right) \cos \theta \\
w & =A \operatorname{Re}\left(w^{\prime}(r) \mathrm{e}^{-\mathrm{i} \omega t}\right) \sin \theta
\end{aligned}
$$

where $u^{\prime}(r), v^{\prime}(r)$ and $w^{\prime}(r)$ are the eigenfunctions for the Orr-Zommerfeld equation associated with the given value of the disturbance frequency $\omega, R$ is the pipe radius, $A$ is the disturbance amplitude. The disturbance frequency $\omega$ was chosen equal to $0.36 u_{0} / R$, and the velocity $u_{0}$ and the pipe radius $R$ corresponded to the Reynolds number equal to 4000 . At initial instant the Poiseuille 

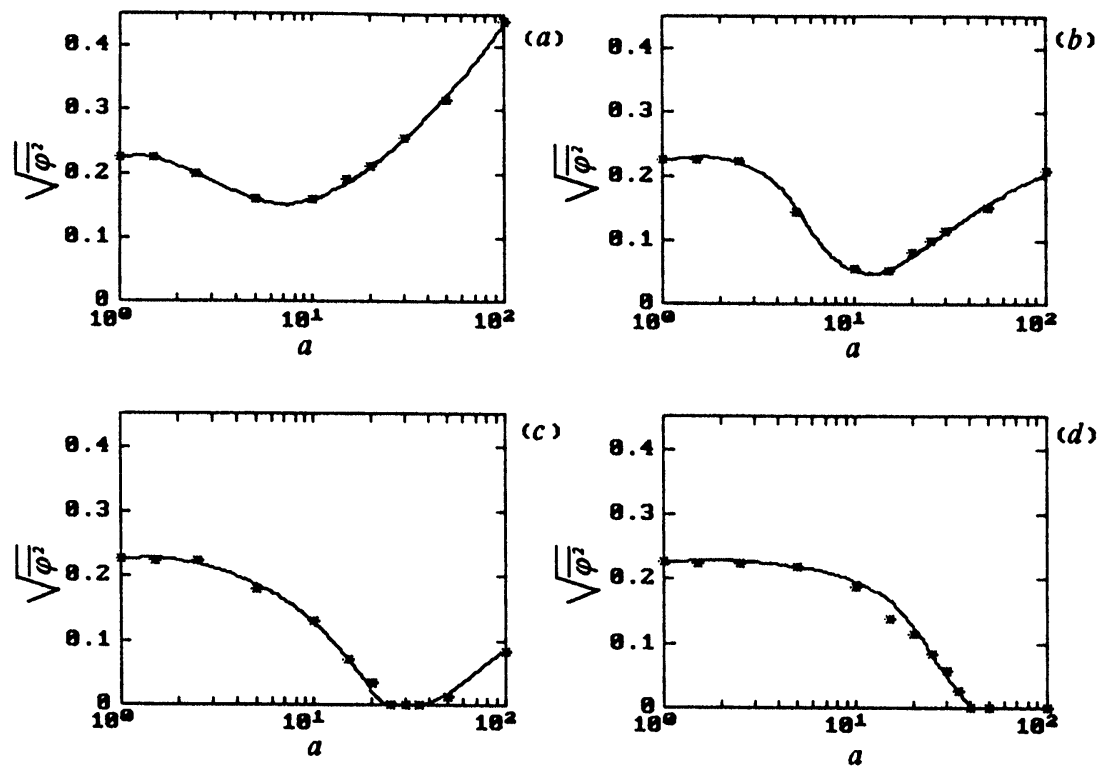

FIGURE 5 The dependencies of $\bar{\varphi}^{1 / 2}$ on $a$ for $\omega_{0}=1, \beta=0.1, \alpha=100, \kappa(2) / \kappa_{\mathrm{cr}}(2)=5.6$ (a) $\omega_{a}=3.5$, (b) $\omega_{a}=6$, (c) $\omega_{a}=11$, and (d) $\omega_{a}=19.75$.

velocity profile was given for whole flow, i.e.

$$
\left.\mathbf{V}\right|_{t=0}=\left\{u_{0}\left(1-\frac{r^{2}}{R^{2}}\right), 0,0\right\}
$$

When the disturbance amplitude $A$ stands out above a certain critical value, after a short time, random high-frequency pulsations appear in the lower part of the pipe from a certain value $x=x_{0}$ onward. The value $x_{0}$ depends only slightly on the distance from the pipe axis $r$, and it is the smaller, the greater is the disturbance amplitude $A$. The occurrence of the turbulent pulsations is accompanied by considerable change of the profile of the mean flow velocity's longitudinal component: it decreases at the pipe axis and increases nearby the pipe wall. The instantaneous distributions of the velocity's longitudinal component in steady regime are shown in Fig. 6 for $A / u_{0}=0.04$; this figure is taken from [37]. If one diminishes progressively the amplitude of the periodic disturbance $A$ then, from a certain its value onward, the turbulence region drifts downstream and the flow in the pipe becomes laminar. As is known (see, for example, [38]), the Poiseuille flow in a circular pipe, as differentiated from the Poiseuille flow in a plane channel, is stable with respect to infinitesimal disturbances for any Reynolds numbers. However, in the case of sufficiently large Reynolds numbers such flow is unstable with respect to disturbances having a finite value. If an attractor associated with turbulence would exist in the system as the disturbance is absent and under the effect of the disturbance the phase trajectory would only come into the attractive domain of this attractor, the turbulence should not disappear when the disturbance resulting in it was taken away. The fact that the turbulence disappears implies that such an attractor is absent. True, it is possible that an attractor comes into existence under the effect of an asynchronous disturbance in itself (see $[39,40]$ ). In this case it has to disappear when the disturbance ceases. Against this possibility are the following facts. Firstly, as is known from the general theory of the asynchronous excitation of oscillations in systems with hard excitation $[39,40]$ such an attractor can appear only within a narrow range of system's 

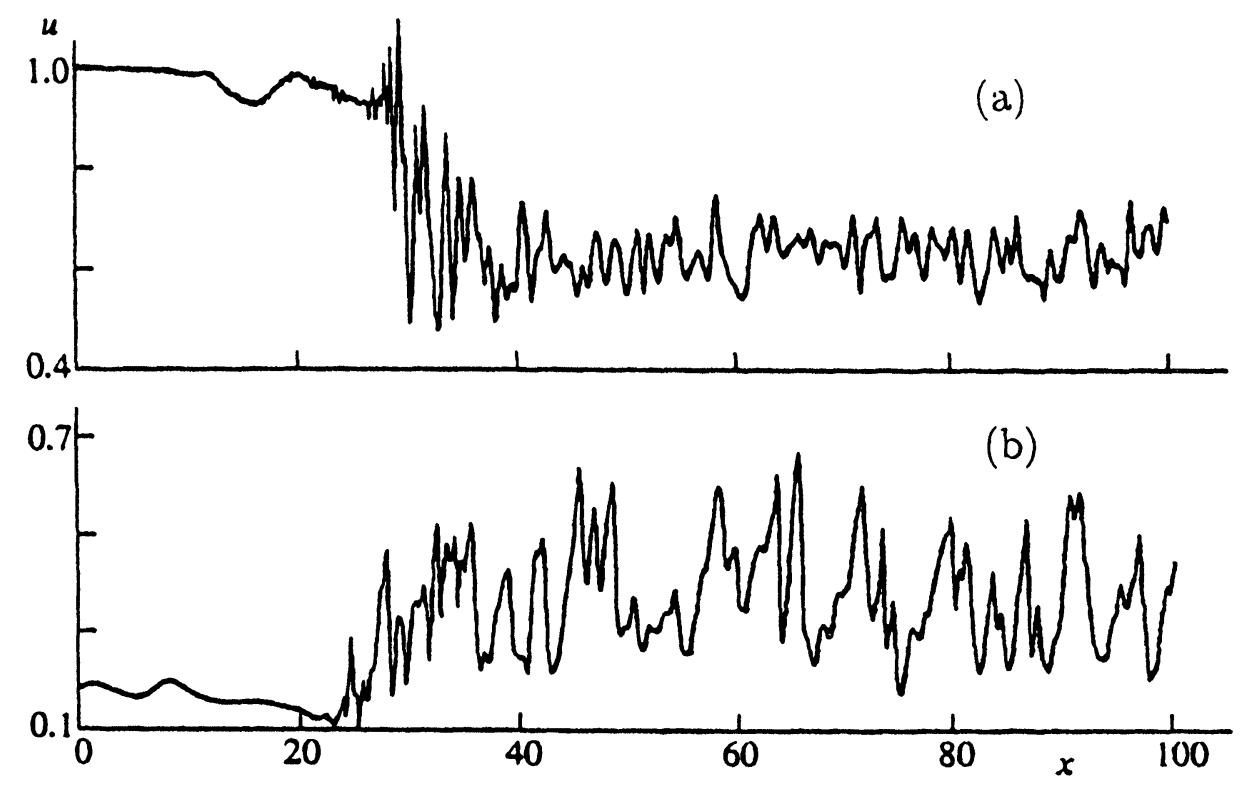

FIGURE 6 Instantaneous distributions of the steady longitudinal velocity component for $A / u_{0}=0.04$ : (a) close to the pipe axis (for $r / R=0.02$ ) and (b) close to the pipe wall (for $r / R=0.93$ ).

parameters, whereas the transition to turbulence was observed by Nikitin over a wide range of Reynolds' numbers. Secondly, asynchronous excitation of self-oscillations, is equally possible both for positive and negative mistunings between the frequencies of the disturbance and self-oscillations, whereas the transition to turbulence was observed only for low disturbance's frequencies. As is seen from the foregoing, such asymmetry is intrinsic just to noise-induced oscillations.

So, the fact that the turbulence disappears, when the disturbance is taken away, implies that the turbulent pulsations resulting from the disturbance are more likely to be noise-induced oscillations than self-oscillations. Therefore it can be conjectured that the turbulence development observed for $A \geq A_{\text {cr }}$ is a result of the noise-induced phase transition bringing into existence an induced attractor. The similarity of the characteristics of turbulence arising in the pipe considered above to the characteristics of turbulence in a pipe with periodic boundary conditions [31], when there is feedback and self-oscillations are excited, counts in favor of this hypothesis. The superficial similarity is illustrated by Fig. 7 constructed from Nikitin's data. If the conjecture made is correct, the role of the periodic disturbance for the development of turbulence reduces only, on the one hand, to ensuring necessary amplification of small fluctuations, which are always present even in numerical experiments, and, on the second hand, to initiating the phase transition, much as is demonstrated with the example of the pendulum's oscillations.

The parallels between turbulent processes and noise-induced pendulum's oscillations can be also traced by the example of the turbulence development and controlling this development in jet flows. It is known [41-43,22] that large-scale turbulence in subsonic jets arises at a certain distance from the nozzle inside the boundary layer whose thickness increases almost linearly with this distance. The onset of the turbulence is accompanied by change of the mean flow velocity. It is also known $[44,45,22]$ that acoustic action upon a jet in the region of its outflow from the nozzle, depending on the frequency, can either initiate the turbulence development or suppress 

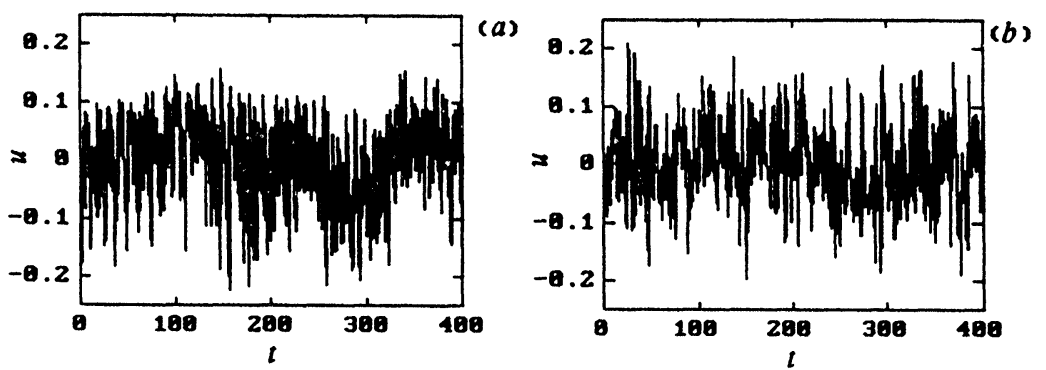

FIGURE 7 The form of turbulent pulsations in a pipe (a) with periodic boundary conditions and (b) with given harmonic disturbance at its inlet.

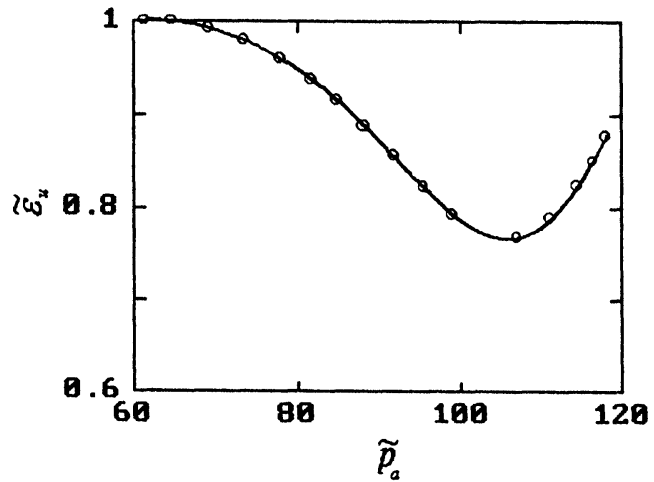

FIGURE 8 The experimental dependence of $\epsilon_{u}$ on the relative amplitude of acoustic pressure $\tilde{p}_{\mathrm{a}}$ measured in decibels for $\mathbf{S t}=2.35, x / D=8$.

it. The initiation occurs at low frequencies of the action, whereas the suppression occurs at high frequencies of the action. Such phenomena were observed experimentally by various investigators. The experimental dependence of the relative root-mean-square pulsation of the longitudinal component of hydrodynamical velocity $\epsilon_{u}$ on the acoustic pressure for $\mathbf{S t}=2.35$ is shown in Fig. 8 taken from [46]. Here $\mathbf{S t}=f_{\mathrm{a}} D / U_{0}$ is the Strouchal number, $f_{\mathrm{a}}$ is the frequency of the acoustic action, $D$ is the nozzle diameter, $U_{0}$ is the mean flow velocity at the jet axis. We see that the turbulent pulsations first decrease as the amplitude of acoustic wave increases and then they increase. This experimental dependence closely resembles one of the dependencies of ${\overline{\varphi^{2}}}^{1 / 2}$ on $a$ shown in Fig. 5 for a pendulum with an addi- tional high-frequency vibration of its suspension axis when the frequency of the action is not too high.

\section{CONCLUSIONS}

The universality of the oscillation theory laws makes it possible to use for the study of complicated concrete systems not only models - "portraits" but far more simple models as well. An example of such a simple model for the elucidation of the character of the turbulent development is considered. It turned out that the excitation of noise-induced oscillations of a pendulum with a randomly vibrating suspension axis and the appearance of turbulent flow in pipes of a finite length and in free subsonic jets have many common features, in spite of perfect distinction between the equations describing these processes.

\section{References}

[1] Mandelshtam, L.I. (1955). Lectures on Oscillations (1930-1932). Collected Works, Vol. 4. Moscow, Izd-vo AN SSSR (in Russian).

[2] Mandelshtam, L.I. (1947). New phenomenon for light scattering. Collected Works, Vol. 1. Moscow, Izd-vo AN SSSR, pp. 293-296; On the light scattering by crystals. Ibid, pp. 305-317 (in Russian).

[3] Mandelshtam, L.I. (1972). Lectures on Optics, Relativity Theory and Quantum Mechanics. Moscow, Nauka (in Russian).

[4] Landa, P.S. and Rosenblum, M.G. (1993). Synchronization and chaotization of oscillations in coupled self-oscillating systems. Appl. Mech. Rev., 46(7), 414-426. 
[5] Blekhman, I.I., Landa, P.S. and Rosenblum, M.G. (1995). Synchronization and chaotization phenomena in oscillatory and rotatory dynamical systems. In: Nonlinear Dynamics: New Theoretical and Applied Results, Ed. J. Awrejcewicz. Akademie-Verlag, Berlin, pp. 17-54.

[6] Blekhman, I.I., Landa, P.S. and Rosenblum, M.G. (1995). Synchronization and chaotization in interacting dynamical systems. Appl. Mech. Rev., 48(11), 733-752.

[7] Neimark, Yu.I. and Landa, P.S. (1987). Stochastic and Chaotic Oscillations. Moscow, Nauka (in Russian); English translation: Kluwer Academic Publishers, Dordrecht-Boston-London, 1992.

[8] Andronov, A.A. (1956). Collected Works. Moscow, Izdvo AN SSSR (in Russian).

[9] Neimark, Yu.I. (1991). Some problems of the qualitative theory of vibrations. Advances in mechanics, 14(3), 87-102.

[10] Landa, P.S. (1996). Nonlinear Oscillations and Waves in Dynamical Systems. Kluwer Academic Publishers, Dordrecht-Boston-London.

[11] Mosekilde, E. and Mouritsen, O.G., Eds. (1995). Modeling the Dynamics of Biological Systems. Springer-Verlag, Berlin.

[12] Gontar, V. (1993). A new theoretical approach to the description of physico-chemical reaction dynamics with chaotic behavior. In: Chaos in Chemistry and Biochemistry, Eds. R.J. Field and L. Györgyi. World Scientific, London, pp. 225-247.

[13] Gribkov, D.A. et al. (1994). Reconstruction of differential equations for auto-stochastic systems from a time series for a single dynamical variable of the process ZhTF, 64(3), pp. 1-12 (in Russian).

[14] Lotka, A.J. (1920). Undamped oscillations derived from the law of mass action. J. Amer. Chem. Soc., 42(8), $1595-1599$.

[15] Volterra, V. (1931). Leçons sur la Theorie Mathematique de la Lutte pour la Vie. Paris, Gauthier-Villars.

[16] Smirnova, O.A. and Stepanova, N.V. (1971). A mathematical model of oscillations in the process of infectious immunity. In: Oscillatory Processes in Biological and Chemical Systems. Puschino-na-Oke, izd. NCBI AN SSSR, pp. 247-251 (in Russian).

[17] Neimark, Yu.I. (1990). A mathematical model of interaction between producers, product and managers. In: $D y$ namics of Systems (Dynamics, Stochasticity, Bifurcations). Gorkiy, Izd-vo GGU, pp. 84-89 (in Russian).

[18] Feigenbaum, M.J. (1978). Quantitative universality for a class of nonlinear transformations. J. Stat. Phys., 19(1), pp. 25-52.

[19] Kaneko, K. (1993). Theory and applications of coupled map lattices, Nonlinear Science: Theory and Applications. Wiley.

[20] Landa, P.S. (1996). Turbulence in nonclosed fluid flows as a noise-induced phase transition. Europhys. Lett., 36(6), pp. 401-406.

[21] Landa, P.S. (1995). What is the turbulence? (Afterword on the paper of Yu.L. Klimontovich) Izv. VUZov, Prikladnaya Nelineynaya Dinamika (2), 37-41 (in Russian).

[22] Ginevsky, A.S. and Landa, P.S. (1995). Excitation of hydrodynamic and acoustic waves in subsonic jet and separation flows. Izv. VUZov, Prikladnaya Nelineynaya Dinamika (2), 42-59 (in Russian).

[23] Landa, P.S. and Zaikin, A.A. (1996). Noise-induced phase transitions in a pendulum with a randomly vibrating suspension axis. Phys. Rev. E, 54(4), pp. 3535-3544.
[24] Landa, P.S. and Zaikin, A.A. (1996). Nonequilibrium noise-induced phase transitions in simple systems. ZhETF, 110(1).

[25] Landa, P.S. and Rosenblum, M.G. (1991). Time series analysis for system identification and diagnostics. Physica $D, 48(1), 232-254$.

[26] Broomhead, D.S. and King, G.P. (1986). Extracting qualitative dynamics from experimental data. Physica $D$, 20(2-3), 217-236.

[27] Schuster, H.G. (1984). Deterministic Chaos. PhysicVerlag, Weinheim.

[28] Manneville, P. (1990). Dissipative Structures and Weak Turbulence. Academic Press, New York.

[29] Rotta, J. (1956). Experimenteller Beitrag zur Entstehung turbulenter Strömung in Rohr. Ing.-Arch., 24(4), 258281.

[30] Townsend, A.A. (1976). The Structure of Turbulent Shear Flow. Cambridge Univ. Press, Cambridge.

[31] Nikitin, N.V. (1994). Direct numerical simulation of three-dimensional turbulent flows in circular pipes. $\mathrm{Me}$ khanika Zhidkosti i Gasa (6), 14-26 (in Russian).

[32] Pomeau, Y. and Manneville, P. (1980). Intermittent transition to turbulence in dissipative dynamical systems. Comm. Math. Phys., 74(2), 189-197.

[33] Platt, N., Spiegel, E.A. and Tresser, C. (1993). On-off intermittency: a mechanism for bursting. Phys. Rev. Lett., 70(3), 279-282.

[34] Pikovsky, A.S. (1984). On the interaction of strange attractors. Z. Phys. B, Condensed Matter, 55, 149-154.

[35] Heagy, J.F., Platt, N. and Hammel, S.M. (1994). Characterization of on-off intermittency. Phys. Rev. E, 49, $1140-1150$

[36] Landa, P.S. and Stratonovich, R.L. (1962). The theory of fluctuational transitions of different systems from one steady state to another. Vestnik $M G U$ (1), 33-45 (in Russian).

[37] Nikitin, N.V. (1995). Spatial approach to numerical simulation of turbulence in pipes. Doklady Akademii Nauk, 343(6), 767-770 (in Russian).

[38] Goldshtik, M.A. and Shtern, V.N. (1977). Hydrodynamic Stability and Turbulence. Novosibirsk, Nauka (in Russian)

[39] Teodorchik, K.F. (1952). Self-Oscillatory Systems. Moscow, Nauka (in Russian).

[40] Landa, P.S. (1980). Self-Oscillations in Systems with Finite Number of Degrees of Freedom. Moscow, Nauka (in Russian).

[41] Ginevsky, A.S. (1969). The Theory of Turbulent Jets and Wakes. Moscow, Mashinostroyeniye (in Russian).

[42] Ho Chih-Ming and Huerre, P. (1984). Perturbed free shear layers. Ann. Rev. Fluid Mech., 16, 365-424.

[43] Belotserkovsky, S.M. and Ginevsky, A.S. (1995). Simulation of Turbulent Jets and Wakes by the Method of Discrete Vortices. Moscow, Nauka (in Russian).

[44] Ginevsky, A.S., Vlasov, E.V. and Kolesnikov, A.V. (1978). Aeroacoustic Interactions. Moscow, Nauka (in Russian).

[45] Hussain, A.K.M.F. and Thompson, C.A. (1980). Controlled symmetric perturbation of the plain jet: an experimental study in the initial region. J. Fluid Mech., 100(2), $397-431$.

[46] Vlasov, Ye.V. and Ginevsky, A.S. (1986). Coherent structures in turbulent jets and wakes. In: VINITI $A N$ SSSR, Itogi Nauki i Tekhniki, Mekhanika Zhidkosti i Gasa, 20, 3-84 (in Russian). 


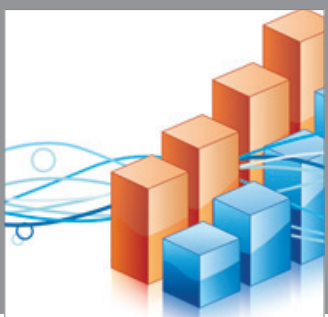

Advances in

Operations Research

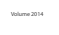

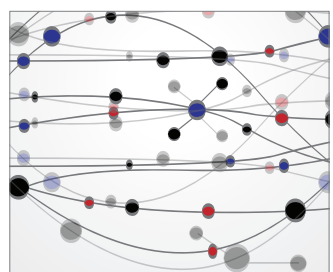

\section{The Scientific} World Journal
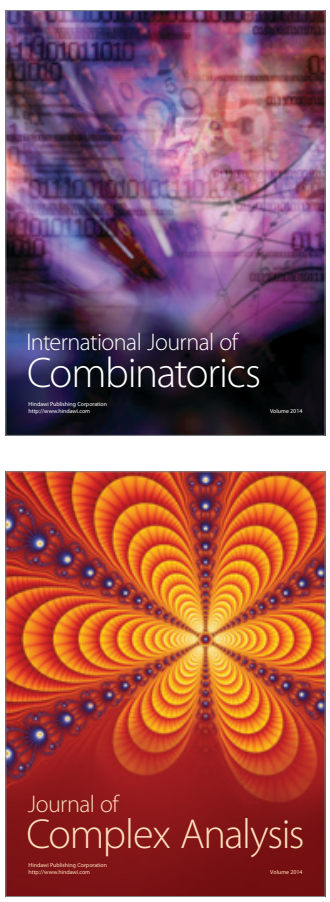

International Journal of

Mathematics and

Mathematical

Sciences
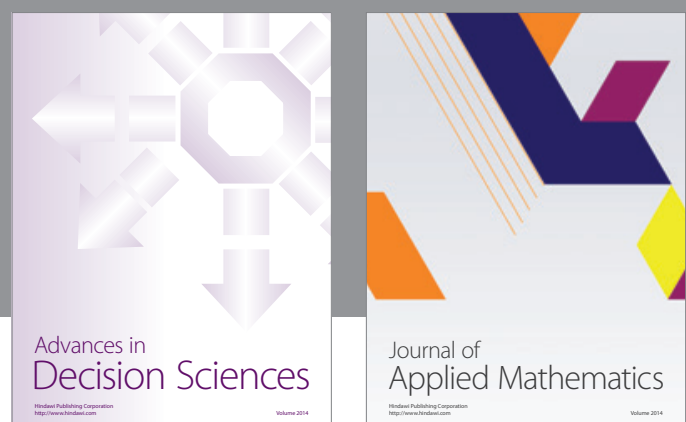

Journal of

Applied Mathematics
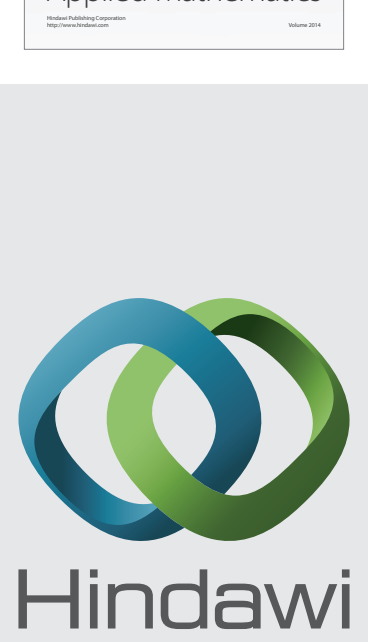

Submit your manuscripts at http://www.hindawi.com
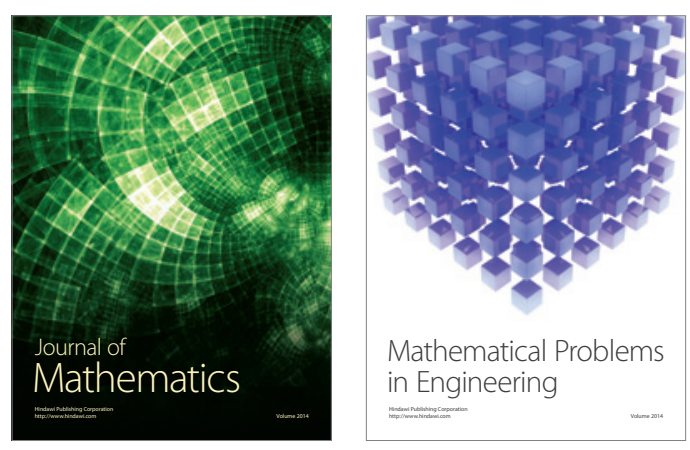

Mathematical Problems in Engineering
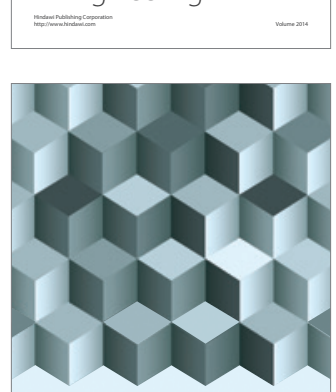

Journal of

Function Spaces
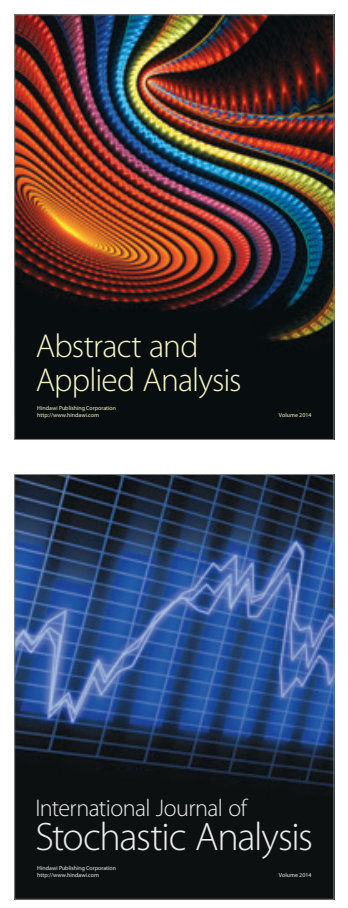

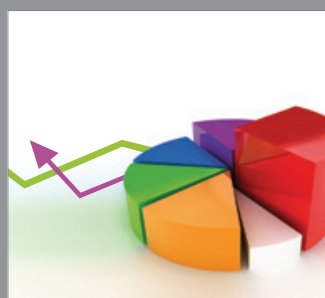

ournal of

Probability and Statistics

Promensencen
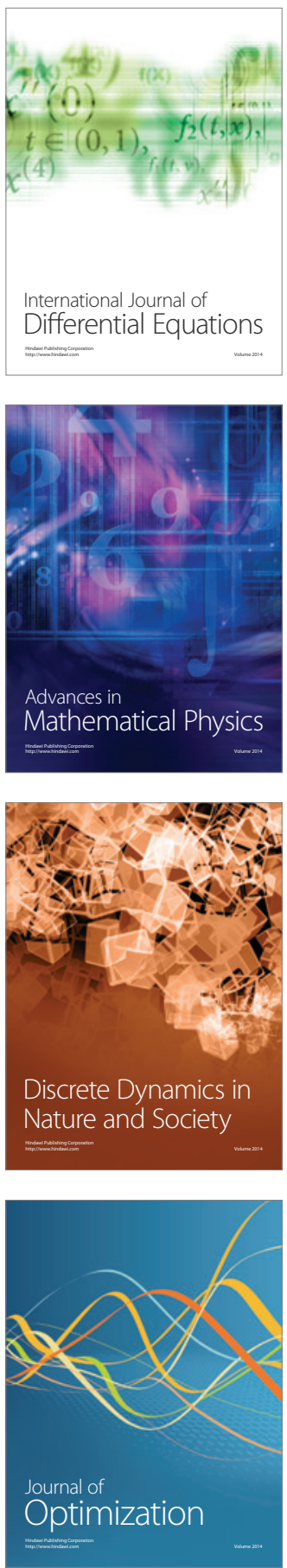